\title{
All-trans retinoic acid effectively reduces atheroma plaque size in a rabbit model of high-fat-induced atherosclerosis
}

\author{
Leila Zarei ${ }^{1, A, B, D, F}$, Mehran Bahrami ${ }^{2, B-D}$, Negin Farhad ${ }^{2, B, D}$, Seyyed Meysam Abtahi Froushani3 ${ }^{3, B, D}$, Ata Abbasi ${ }^{4}, A, C-F$ \\ 1 Solid Tumor Research Center, Urmia University of Medical Sciences, Iran \\ ${ }^{2}$ Student Research Committee, Urmia University of Medical Sciences, Iran \\ ${ }^{3}$ Department of Microbiology, Veterinary Faculty, Urmia University, Iran \\ ${ }^{4}$ Department of Pathology, Faculty of Medicine, Urmia University of Medical Sciences, Iran \\ A - research concept and design; $\mathrm{B}$ - collection and/or assembly of data; $\mathrm{C}$ - data analysis and interpretation; \\ $\mathrm{D}$ - writing the article; $\mathrm{E}$ - critical revision of the article; $\mathrm{F}$ - final approval of the article
}

\section{Address for correspondence \\ Ata Abbasi}

E-mail: aabbasi@alumnus.tums.ac.ir

\section{Funding sources}

Research grant No. 1886 from Urmia University

of Medical Sciences, Iran

Conflict of interest

None declared

Received on December 9, 2016

Reviewed on May 5, 2017

Accepted on June 7, 2017

D0I

10.17219/acem/74552

\section{Copyright}

Copyright by Author(s)

This is an article distributed under the terms of the

Creative Commons Attribution Non-Commercial License

(http://creativecommons.org/licenses/by-nc-nd/4.0/)

\begin{abstract}
Background. Atherosclerosis (AS) is one of the most prevalent causes of death around the world. Since there are different types of risk factors, different types of medications focus on preventing atheromas and plaques from establishing or on preventing established plaques from growing.

Objectives. The aim of this study was to evaluate the effect of all-trans retinoic acid (atRA) on AS in a rabbit model of fat-induced AS.

Material and methods. Atherosclerosis was induced by a high-fat diet (HFD) for 75 days. Thirty rabbits were randomly divided into 5 groups. Group 1 was the negative control group and received a normal diet. The animals in the other groups were fed a HFD. Group 2 (the AS positive control group) received no drugs, Group 3 received atorvastatin orally ( $20 \mathrm{mg} / \mathrm{kg} / \mathrm{day})$, Group 4 received atRA ( $5 \mathrm{mg} / \mathrm{kg} / \mathrm{day}$, orally), and Group 5 received both drugs. All medications were started on day 45 and continued until the end of the study. Fasting blood samples were obtained for lipid profile evaluation. The aorta sections were evaluated for maximum wall and intima thickness.
\end{abstract}

Results. Oral administration of atRA, atorvastatin or their combination significantly improved serum lipid profile $(p<0.001)$. Atorvastatin and atRA significantly decreased serum total cholesterol and LDL-cholesterol levels in HFD ( $p<0.001)$. No difference was found in serum HDL-cholesterol levels among the studied groups. The HFD group (Group 2 - positive control) showed significant intima irregularities with fat deposition and foamy macrophage accumulation (atheroma). Administration of atRA and atorvastatin significantly decreased the size of atherosclerotic plaques (intima thickness). The maximum vessel wall and intima thickness were significantly decreased after atRA and atorvastatin administration $(p<0.001)$. No difference was found between atRA and atorvastatin effectiveness, but combination therapy significantly decreased AS size in comparison to using either of the drugs alone $(p<0.001)$.

Conclusions. In reducing AS plaque size, atRA is as effective as atorvastatin. Additionally, the combination therapy of atRA and atorvastatin decreased AS size much more effectively, showing their synergistic effect. atRA can also improve the serum lipid profile.

Key words: atherosclerosis, high-fat diet, all-trans retinoic acid 


\section{Introduction}

Atherosclerosis (AS) is one of the most prevalent causes of death around the world. ${ }^{1}$ The formation and progression of atherosclerosis is a long-term process which begins with an accumulation of fat within the vessel walls. The fat accumulation triggers an inflammatory process as well as platelet activation and accumulation, which subsequently leads to the formation of an atherosclerotic plaque. ${ }^{2,3}$ There are some traditional risk factors involved in the initiation or progression of plaques. Hypertension, smoking, diabetes mellitus (DM), obesity, hyperlipidemia, and infections are some well-known risk factors. ${ }^{1,4}$ Since there are different types of risk factors, different types of medications are used, but most of therapy methods have focused on preventing atheromas and plaques from establishing and on preventing established plaques from growing.

All-trans retinoic acid (atRA) is derived from vitamin A through an enzymatic process. ${ }^{5}$ It has antioxidant activity and is involved in cellular development, growth and differentiation. ${ }^{6-8}$ It is also known that atRA acts as an anti-inflammatory agent and can inhibit platelet function. ${ }^{2}$ Recent studies have uncovered some protective effects of atRA on the cardiovascular system. ${ }^{9,10}$ It has also been shown that atRA and its derivatives can inhibit DM development, and decrease body weight and lipid production by regulating fat metabolism in various organs. ${ }^{5,9-12}$ Considering the effects of atRA on DM and lipid metabolism, as well as its anti-inflammatory and anti-platelet activation effect, we hypothesized that atRA can be useful in the treatment of AS.

The aim of this study was to evaluate the effect of atRA on AS in a rabbit model of fat-induced AS and to compare the results with atorvastatin as a control drug.

\section{Material and methods}

\section{Animals}

Thirty male New Zealand rabbits were included in the present study. All animals were housed for 1 week in a temperature- and humidity-controlled vivarium $\left(22 \pm 2^{\circ} \mathrm{C}, 30-60 \%\right)$ with a light/dark cycle of $10 \mathrm{~h} / 14 \mathrm{~h}$ and access to a standard diet and water.
All animal procedures were performed in accordance with the Guide for the Care and Use of Laboratory Animals (National Institutes of Health (NIH) US publication No. 85-23, revised 1985). All experiments were performed in agreement with the ethical considerations recommended by the Pasteur Institute of Iran in Tehran, and the study protocol was reviewed and approved by the Ethical Committee of the Urmia University of Medical Sciences, Iran.

\section{Drug administration}

The rabbits were randomly divided into 5 groups. Each group contained 6 rabbits. Group 1 was treated as a negative control group and was fed a normal diet for 75 days. The animals in Groups 2-5 were fed a high-cholesterol (fat) diet (HFD; $1 \%$ of body weight) for 75 days. Group 2 served as the AS positive control group and was not treated with any drugs, Group 3 received atorvastatin orally ( $20 \mathrm{mg} / \mathrm{kg} /$ day) starting on day 45 for 30 days (until the end of the study), Group 4 received atRA ( $5 \mathrm{mg} / \mathrm{kg} /$ day, orally) starting on day 45 for 30 days (until the end of the study), and Group 5 received both atorvastatin $(10 \mathrm{mg} / \mathrm{kg} /$ day $)$ and atRA $(2.5 \mathrm{mg} / \mathrm{kg} /$ day $)$ starting on day 45 for 30 days (until the end of the study).

At the end of the experiment, the rabbits fasted for $12 \mathrm{~h}$ prior to anesthesia and peripheral blood samples were obtained from the marginal vein of their ear. Then, serum samples were separated and analyzed for total cholesterol (TC), triglyceride (TG), high-density lipoprotein (HDL), and low-density lipoprotein (LDL) counts. Finally, the rabbits were sacrificed and the aorta of each rabbit was carefully resected as previously described. ${ }^{13}$

\section{Biochemical measurement}

Blood samples were centrifuged at $3000 \mathrm{rpm}$ for $10 \mathrm{~min}$ and serum samples were separated. Serum levels of TC, TG, LDL, and HDL were measured using an autoanalyzer (BT 4500; Biotecnica Instruments, Rome, Italy).

\section{Tissue preparation}

The aorta, including the ascending and descending parts, was excised and totally immersed in phosphate buffered formalin (PBF) for at least $24 \mathrm{~h}$ for fixation. After

Table 1. Serum lipid levels in the studied groups [mg/dL]

\begin{tabular}{|l|c|c|c|c|c|c|c|c|}
\multicolumn{1}{|c|}{ Serum } & $\begin{array}{c}\text { Group 1 } \\
\text { (negative control) }\end{array}$ & $\begin{array}{c}\text { Group 2 } \\
\text { (positive control) }\end{array}$ & $\begin{array}{c}\text { Group 3 } \\
\text { (atorvastatin) }\end{array}$ & $\begin{array}{c}\text { Group 4 } \\
\text { (atRA*) }\end{array}$ & $621 \pm 259$ & $153.8 \pm 61$ \\
(atRA + atorvastatin)
\end{tabular}

TC - total cholesterol; TG - triglyceride; HDL - high-density lipoprotein; LDL - low-density lipoprotein; * atRA - all-trans retinoic acid;

** $p$-value $<0.05$ is significant. 


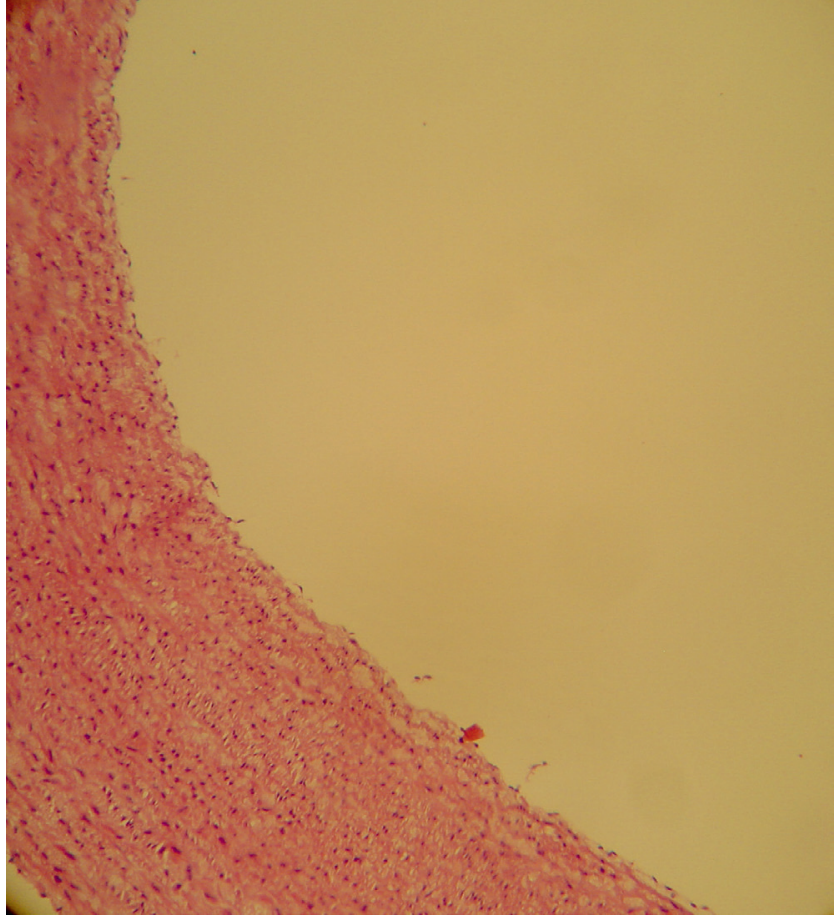

Fig. 1A. Aortic tissue (muscular wall) of a rabbit not receiving a high-fat diet (HFD) (negative control - Group 1) (hematoxylin and eosin (H\&E) staining, $\times 10$ magnification)

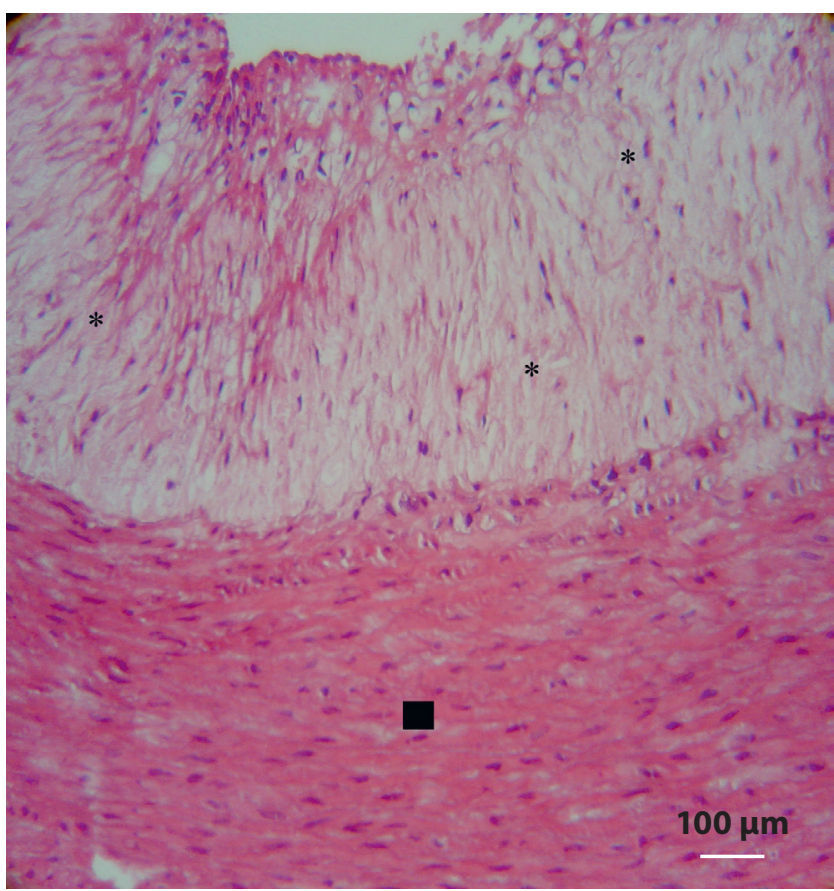

Fig. 1B. Aortic tissue of a rabbit receiving a high-fat diet (HFD) without any drug administration (positive control - Group 2). A large atherosclerotic plaque composed of foamy macrophages (clearly stained area marked with *) is seen attached to the vessel wall (marked with a black square) (hematoxylin and eosin (H\&E) staining, $\times 40$ magnification)

fixation, the samples were embedded in paraffin. Then, $5 \mu \mathrm{m}$ sections were obtained from each paraffin block and stained with hematoxylin and eosin (H\&E). The maximum wall thickness of the aorta and the intima thickness

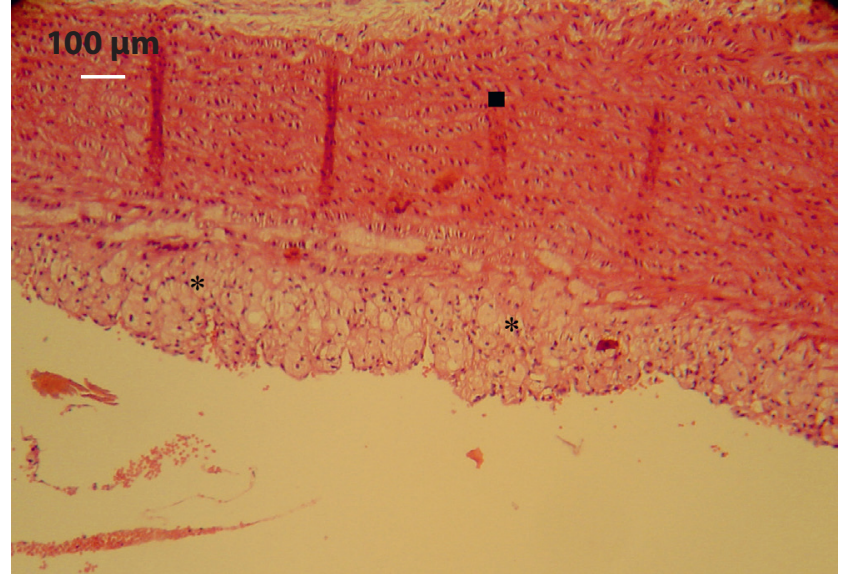

Fig. 1C. Aortic tissue of a rabbit treated with atorvastatin (Group 3), with smaller atherosclerotic plaque (area marked with *) compared to the positive control (Group 2) in Fig. 2. The vessel wall is marked with a black square (hematoxylin and eosin (H\&E) staining, $\times 20$ magnification)

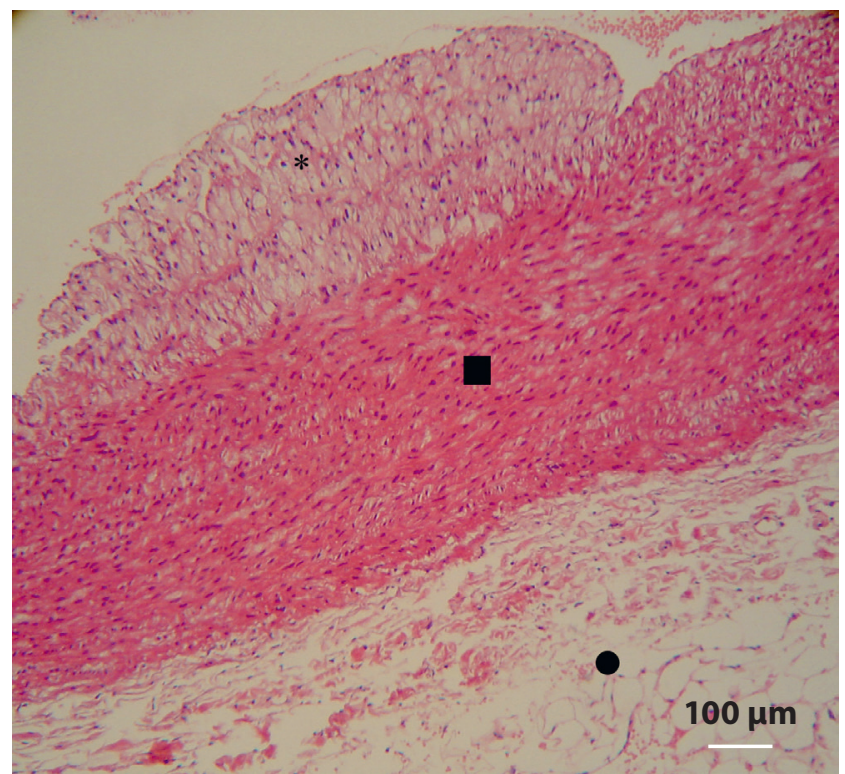

Fig. 1D. Aortic tissue of a rabbit receiving atRA (Group 4), with smaller atherosclerotic plaque (area marked with *), compared to the positive control (Group 2) in Fig. 2. The vessel wall is marked with a black square. The black circle shows the vessel serosa (hematoxylin and eosin (H\&E) staining, $\times 20$ magnification)

(plaque size) of each sample were measured using an image evaluation program (Optika, Vittorio Veneto, Italy) and were compared with other samples.

\section{Statistical analysis}

The results are expressed as mean \pm standard error of the mean (SEM). Statistical analysis was performed using SPSS v. 16.0 (SPSS Inc., Chicago, USA). Statistical evaluation of the data was performed using an analysis of variance (ANOVA). The normality of data was evaluated with the Kolmogorov-Smirnov test; $\mathrm{p}$-values $<0.05$ were considered statistically significant. 


\section{Results}

\section{Serum lipid profile}

Levels of LDL, HDL, TG, and TC were significantly higher in the HFD positive control group (Group 2) compared to the negative control group (Group 1) (p < 0.001). Our data showed that oral administration of atRA or atorvastatin, or their combination, significantly decreased TC and LDL serum levels ( $\mathrm{p}<0.001)$. Additionally, we found that atorvastatin reduced serum TC and LDL levels more effectively than atRA ( $p<0.001)$, but their combination therapy showed no significant difference in effectiveness compared to atorvastatin alone $(\mathrm{p}=0.21)$. No statistical difference was found in serum HDL levels among the studied groups. The details of the serum lipid profiles are included in Table 1.

\section{Histopathological evaluation}

The sample from each rabbit's aorta was evaluated using a light microscope (Olympus BH-21; Olympus, Tokyo, Japan). In the negative control group no atheromas were identified, but the HFD group (Group 2 - positive control) showed significant irregularities on the inner surface of the aorta (intima), with fat deposition and foamy macrophage accumulation (atheroma). Oral administration of atRA and atorvastatin significantly decreased the size of the atherosclerotic plaques and intima thickness (Fig. 1A-D, 2). The maximum vessel wall and intima thickness were measured in each group and showed a significant decrease after atRA, atorvastatin or their combined administration $(\mathrm{p}<0.001)$. No difference was found between atRA and atorvastatin in reducing AS size, but the combination therapy of atRA and atorvastatin significantly decreased AS size in comparison to using either drug alone (p < 0.001), (Fig. 3A, 3B).

\section{Discussion}

Derived from vitamin A, atRA is involved in some cell regulatory processes, including cell migration, healing and differentiation. ${ }^{2,13}$ Recent studies have revealed the effects of atRA on the cardiovascular system. It was shown that atRA can inhibit restenosis of a coronary artery after angioplasty. ${ }^{14}$ In this study, we demonstrated that oral administration of atRA in a rabbit model of fat-induced AS improved the serum lipid profile and also decreased the size of atherosclerotic plaques. We observed similar results with atorvastatin, which was used in our study as a control drug. Atorvastatin is one of the main drugs widely used to lower serum lipids levels in clinical practice. ${ }^{9,15-17}$

In this study, we found atRA very effective in decreasing the size of AS. Although we found no superiority for atRA over atorvastatin in reducing AS, there are some studies

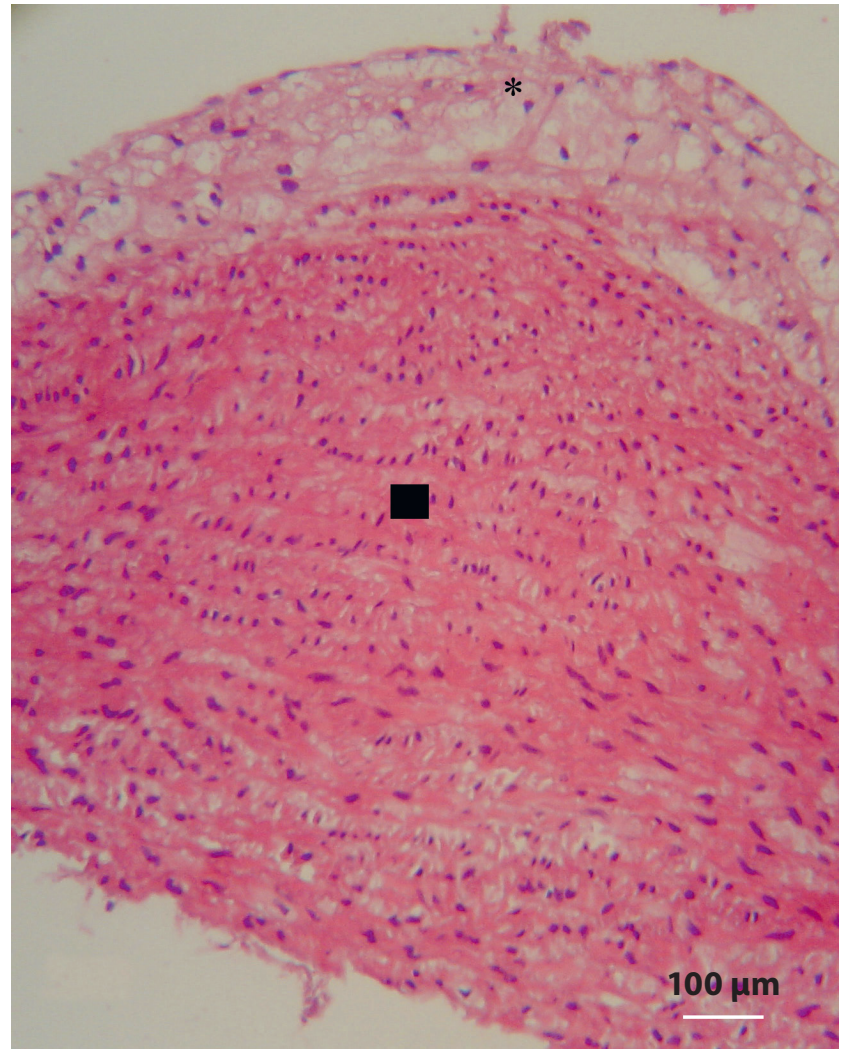

Fig. 2. Aortic tissue of a rabbit receiving both atorvastatin and atRA (Group 5). There is a small plaque (area marked with *) attached to the vessel wall (area marked with a black square) (hematoxylin and eosin (H\&E) staining, $\times 40$ magnification)

which have shown that atRA is much more effective than atorvastatin. ${ }^{2}$ The benefits of atRA can be related to the following mechanisms: reducing lipotoxicity-induced oxidative stress, inhibiting the production of reactive oxygen species (ROS), enhancing fatty acid oxidation, activating macrophages, reducing inflammation, and inhibiting coagulatory factors. ${ }^{2,18-22}$ As a novel finding, we found that combination therapy of atRA and atorvastatin decreased AS size more effectively than administering either one alone. These findings suggest that atRA and atorvastatin act through different mechanisms and that their combination therapy has a synergistic effect on AS treatment.

We also found that atRA can reduce serum TC and LDL-cholesterol levels, although we found atorvastatin to be more effective in reducing serum TC and LDLcholesterol levels than atRA. We also found no difference between atorvastatin and its combination therapy with atRA in lowering serum TC and LDL-cholesterol levels. These findings are in line with other studies on this topic. ${ }^{2}$ We also found no differences in serum HDL-cholesterol levels in the compared groups.

Our study had some limitations. We did not explore the underlying mechanisms through which atRA could reduced atheroma size or serum lipid levels. Some studies have explored the role of inflammation and stated that atherosclerosis is an inflammatory process. ${ }^{23}$ Others have shown that macrophage activation by retinoic acid 


\section{maximum vessel wall thickness $[\mu \mathrm{m}]$}

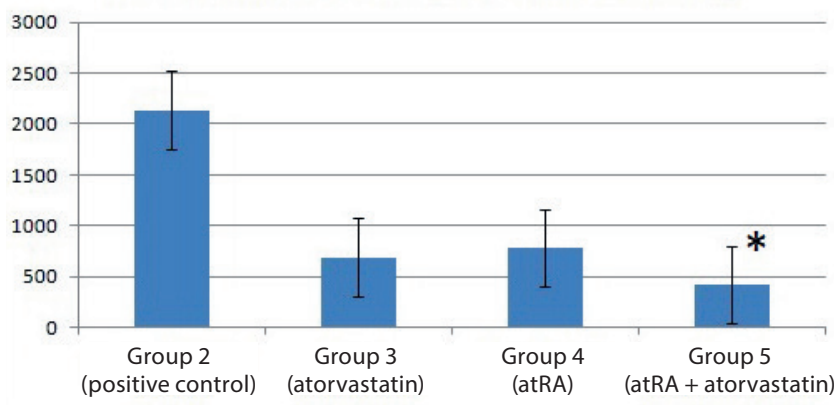

Fig. 3A. Aortic maximal wall thickness in rabbits. The wall thickness analysis showed that all-trans retinoic acid (atRA) and atorvastatin can both reduce the wall thickness and that their combination therapy had the most effective results $(p<0.001)$. There was no difference in effectiveness between atRA and atorvastatin, but as this figure shows $(p=0.3)$, combination therapy showed a significant difference in effectiveness with both atRA and atorvastatin $\left({ }^{*} p<0.001\right)$

intima thickness $[\mu \mathrm{m}]$

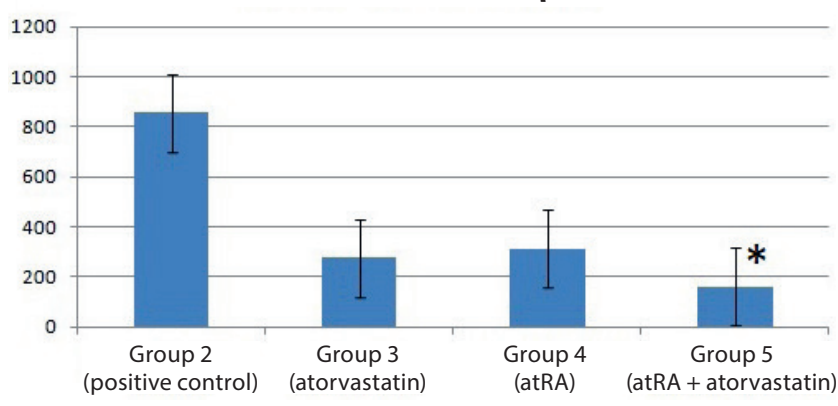

Fig. 3B. Aortic intimal thickness. The results were similar to wall thickness analysis as both all-trans retinoic acid (atRA) and atorvastatin significantly decreased the intima thickness $(p<0.001)$. Combination therapy decreased the intima thickness more effectively than either of the drugs alone $\left({ }^{*} p<0.001\right)$. No difference in effectiveness was found between atRA and atorvastatin $(p=0.24)$

receptors, which leads to improved antiapoptotic activity of the macrophages, can increase removal of apoptotic cells in AS plaques. ${ }^{20}$ In another study by Bilbija et al., an overexpression of retinoic acid target genes was shown in coronary artery disease (CAD). ${ }^{24}$ Bilbija et al. also reported that atRA has antiproliferative effects on cardiomyocytes and cardiofibroblasts and concluded that the antiproliferative effects of atRA may be beneficial because it may be used to delay cardiomyocyte remodeling, reduce restenosis and preserve the functions of the cardiovascular system. ${ }^{24}$ But in none of the related studies are the underlying signal transduction pathways, which lead to AS reduction and inhibition, clearly defined.

We found that atRA can reduce AS plaque size as effectively as atorvastatin. Additionally, their combination therapy improved their power and decreased AS size much more effectively. Our study is a novel experiment which studied the combination effect of atRA and atorvastatin on both AS and serum lipid profile. This combination effect has not been evaluated in any other study. We found that combined administration of atRA and atorvastatin had a synergistic effect only in reducing AS plaque size, but not in lowering serum lipids levels. This finding may suggest a mechanism other than lowering serum lipids levels in reducing AS plaque size. We also showed a lipid-lowering effect for atRA, although it was less effective than atorvastatin. Our results nominate atRA as a potential therapeutic agent in the treatment of AS and subsequent CAD. We suggest performing further studies to find out other therapeutic and adverse effects of atRA, as well as its mechanism of action, the optimal dosage and the route of administration. This may lead to its use in a combination therapy with statins in patients with CAD to better control the disease and reduce ischemic attacks in future.

\section{References}

1. Rezaee-Zavareh MS, Tohidi M, Sabouri A, Ramezani-Binabaj M, Sadeghi-Ghahrodi M, Einollahi B. Infectious and coronary artery disease. ARYA Atheroscler. 2016;12(1):41-49.

2. Zhou B, Pan Y, Hu Z, et al. All-trans-retinoic acid ameliorated high fat diet-induced atherosclerosis in rabbits by inhibiting platelet activation and inflammation. J Biomed Biotechnol. 2012;2012:259693.

3. Lusis AJ. Atherosclerosis. Nature. 2000;407(6801):233-241.

4. Talavera-Garcia E, Delgado-Lista J, Garcia-Rios A, et al. Influence of obesity and metabolic disease on carotid atherosclerosis in patients with coronary artery disease (CordioPrev Study). PLoS One. 2016;11(4):e0153096.

5. Pellegrini C, Columbaro M, Capanni C, et al. All-trans retinoic acid and rapamycin normalize Hutchinson Gilford progeria fibroblast phenotype. Oncotarget. 2015;6(30):29914-29928.

6. Swift J, Ivanovska IL, Buxboim A, et al. Nuclear lamin-A scales with tissue stiffness and enhances matrix-directed differentiation. Science. 2013;341(6149):1240104.

7. Jiang SJ, Campbell LA, Berry MW, Rosenfeld ME, Kuo CC. Retinoic acid prevents Chlamydia pneumoniae-induced foam cell development in a mouse model of atherosclerosis. Microbes Infect. 2008;10(12-13): 1393-1397.

8. Chambon P. A decade of molecular biology of retinoic acid receptors. FASEB J. 1996;10(9):940-954.

9. Guleria RS, Singh AB, Nizamutdinova IT, et al. Activation of retinoid receptor-mediated signaling ameliorates diabetes-induced cardiac dysfunction in Zucker diabetic rats. J Mol Cell Cardiol. 2013;57:106-118.

10. Van $\mathrm{YH}$, Lee $W H$, Ortiz S, Lee $M H$, Qin HJ, Liu CP. All-trans retinoic acid inhibits type-1 diabetes by $T$ regulatory (Treg)-dependent suppression of interferon-gamma-producing T-cells without affecting Th17 cells. Diabetes. 2009;58(1):146-155.

11. Amengual J, Ribot J, Bonet ML, Palou A. Retinoic acid treatment increases lipid oxidation capacity in skeletal muscle of mice. Obesity. 2008;16(3):585-591.

12. Berry DC, Noy N. All-trans-retinoic acid represses obesity and insulin resistance by activating both peroxisome proliferation-activated receptor beta/delta and retinoic acid receptor. Mol Cell Biol. 2009;29(12):3286-3296.

13. Benson MJ, Pino-Lagos K, Rosemblatt M, Noelle RJ. All-trans retinoic acid mediates enhanced $T$ reg cell growth, differentiation, and gut homing in the face of high levels of co-stimulation. J Exp Med. 2007;204(8):1765-1774.

14. Herdeg C, Oberhoff M, Baumbach A, et al. Effects of local all-transretinoic acid delivery on experimental atherosclerosis in the rabbit carotid artery. Cardiovasc Res. 2003;57(2):544-553.

15. Tehrani S, Mobarrez F, Antovic A, et al. Atorvastatin has antithrombotic effects in patients with type 1 diabetes and dyslipidemia. Thromb Res. 2010;126(3):e225-231.

16. Chockalingam P, Vinayagam NS, Chockalingam V, Chockalingam A. Remarkable regression of coronary atherosclerosis: An interplay of pharmacotherapeutic and lifestyle factors. Ind Heart J. 2016;68(2):188-189.

17. Puri $\mathrm{R}$, Nissen $\mathrm{SE}$, Ballantyne CM, et al. Factors underlying regression of coronary atheroma with potent statin therapy. Eur Heart $J$. 2013;34(24):1818-1825. 
18. Nissen SE, Nicholls SJ, Sipahi I, et al; ASTEROID Investigators. Effect of very high-intensity statin therapy on regression of coronary atherosclerosis: The ASTEROID trial. JAMA. 2006;295(13):1556-1565.

19. Amengual J, Ribot J, Bonet ML, Palou A. Retinoic acid treatment increases lipid oxidation capacity in skeletal muscle of mice. Obesity (Silver Spring). 2008;16(3):585-591.

20. Rebe C, Raveneau M, Chevriaux A, et al. Induction of transglutaminase 2 by a liver $X$ receptor/retinoic acid receptor alpha pathway increases the clearance of apoptotic cells by human macrophages. Circ Res. 2009;105(4):393-401.
21. Imaizumi T, Yagihashi N, Kubota K, et al. Expression of retinoic acidinducible gene-I (RIG-I) in macrophages: Possible involvement of RIG-I in atherosclerosis. J Atheroscler Thromb. 2007;14(2):51-55.

22. Kim YM, Kim JH, Park SW, Kim HJ, Chang KC. Retinoic acid inhibits tissue factor and HMGB1 via modulation of AMPK activity in TNF-a activated endothelial cells and LPS-injected mice. Atherosclerosis. 2015;241(2):615-623.

23. Ross R. Atherosclerosis - an inflammatory disease. N Engl J Med. 1999;340(2):115-126.

24. Bilbija D, Elmabsout AA, Sagave J, et al. Expression of retinoic acid target genes in coronary artery disease. Int J Mol Med. 2014;33(3):677-686. 